\author{
Assistant Professor Muhammad SHERAZ, PhD \\ E-mail: msheraz@iba.edu.pk \\ Department of Mathematical Sciences, School of Mathematics and \\ Computer Science \\ Institute of Business Administration Karachi, Pakistan \\ Imran NASIR, PhD student \\ E-mail: imrannasir.07435@iba.khi.edu.pk \\ Department of Mathematical Sciences, School of Mathematics and \\ Computer Science \\ Institute of Business Administration Karachi, Pakistan \\ Associate Professor Silvia DEDU, PhD \\ E-mail: silvia.dedu@csie.ase.ro \\ Department of Applied Mathematics \\ The Bucharest University of Economic Studies
}

\title{
EXTREME VALUE ANALYSIS AND RISK ASSESSMENT: A CASE OF PAKISTAN STOCK MARKET
}

\begin{abstract}
The aim of this paper consists in developing a univariate extreme value analysis, with applications to the Pakistan Stock Exchange (PSX-100). The main focus refers to assessing the risk and statistical properties regarding the tails of the fitted heavy-tails distributions. For this purpose, we implement generalized extreme value distribution (GEV) and generalized Pareto distribution (GPD) by following the block maxima approach, peak-over threshold (POT) method and Poisson processes to several declustered periods. PSX-100 has become the emerging and best performer financial market in the south-Asian region in the last decade. Therefore, statistical properties of extreme events of the stock market have significant importance for investors. We have also addressed the modelling of Value-at-Risk (VaR) and Expected-Shortfall (ES) risk measures, in the context of extreme value theory (EVT).

Keywords: Extreme value theory, Tail events, Risk management, Value-atRisk, Expected-Shortfall.

JEL Classification : C00, C1, E4, E5, G1, G2

\section{Introduction}

In traditional financial markets, several factors such as crashes of stock markets, currency crashes and other extreme events cause immense losses for investors. In risk management, quantification of the probability of rare and extreme
\end{abstract}


events has significant importance. Therefore, the choice of the appropriate probability distribution of asset returns depends on the stability of the financial market. In this regard, the statistical properties of the asset return distribution play a vital role for investors and risk managers. In risk-modelling, empirical fat-tailed returns distributions are frequently encountered, and properties of the tails of these distributions have the utmost importance. Hence, based on the above discussion, it is valuable to investigate the tail behaviors of the asset returns distribution of emerging financial markets of the developing countries. In this paper, we aim to study the emerging and best performing south-Asian stock market of Pakistan in the context of extreme value theory. The PSX-100 share index (formally Karachi Stock Exchange, KSE) emerged as the integration of three local stock exchanges under the promulgation of the new Securities Act, 2015. PSX-100 has shown a significant rise and reached historic levels after the unification. In 2016, PSX-100 was declared an emerging Asian market. Pakistan's qualification for the Morgan Stanley Capital International (MSCI) Emerging market index in 2017 resulted in a record high of 49,876 base points, which is unbroken to this day. Pakistan Stock Exchange had been named Asia's best performer in 2016.

In 2020, a New York based global markets research firm, marketcurrentswealthnet.com (accessed on 27 April 2021), published that PSX has become the best performer in Asia and the world's fourth-best performing market. Pakistan's stock market is an extremely sensitive and highly volatile market. It reacts quickly to political news, government policies and media developed stories. On the other hand, rapid growth after unexpected returns is the typical behavior of the market. The dynamics of price movement in a stock market depends on different factors, such as monetary policy, the impact of interest rates, and the promulgation of unfavorable news. Therefore, we choose to investigate the PSX100 index and characterize its statistical properties using the Extreme Value Theory (EVT) and information theory. To our best knowledge, very few studies have been conducted on PSX-100 using EVT. We aim to fill the existing research gap on the most emerging and best performer market of PSX-100 in Asia.

\section{Literature Review}

Fuller was probably the first who introduced the applications of extreme value distribution in 1914. Bortkiewicz (1922) developed the modern EVT, and later, Fisher and Tippett (1928) set down the foundations of the asymptotic theory of the distributions of extreme values. Hill (1975) studied a general approach to investigate inferences and properties of the tail of a distribution. For more thorough details on EVT, the reader is referred to Leadbetter et al. (1983), Beirlant et al. (2004) and McNeil et al. (2005). Thereafter, several researchers have provided various applications of extreme value distributions. See, for example, Malevergne et al. (2006), Goncu et al. (2012), Sing et al. (2017). The stock return distributions in emerging and developing markets show more leptokurtic behaviour as compared to developed economies. Therefore, returns distribution and other financial assets, generally, exhibit fat-tail behaviour. Mandelbrot (1963) introduced a fat-tail 
distribution model of price behaviour in speculative markets. The model replaced the Gaussian distribution with another family of probability distributions referred to as stable Paretian. Rama Cont (2001) investigated some facts emerging from the statistical analysis of price variations in various financial markets. This study suggests that stylized statistical properties of a financial asset may include heavy tail. Sheraz and Dedu (2020) studied stochastic models of fat-tail returns and riskmodelling to investigate the returns distribution of digital currencies. Several studies exist on application of EVT for stock markets crashes and periods of high volatility. For example, Gencay and Selcuk (2004) studied few Asian markets in the context of VaR and EVT. Djakovic et al. (2011) tested the performance of the EVT on daily returns of four different stock markets. Uppal and Mudakkar (2014) studied the case of the Pakistani market and employed the VaR measure based on EVT. Bozovic and Totic (2015) examined the left tail behaviour of the returns on the several European stock markets and emphasized the importance of the extreme events. Other approaches, based on information measures were used by Sheraz and Nasir (2021), Sheraz et al. (2020) and Preda et al. (2016).

This paper is organized as follows. Section 3 discusses the methodology of modelling the extreme events and related framework. In Section 4, we give a statistical overview of the data we have used. Section 5 presents our empirical developments. Section 6 concludes and summarizes our findings.

\section{Methodology}

In this paper, we aim to analyze the distributional behavior of the PSX-100 daily closing returns for extreme events. We investigate the statistical properties of the underlying data by implementing the EVT. It provides analogues of the central limit theorem for the extreme values in a sample.

Suppose $\left(Y_{1}, Y_{2} \ldots, Y_{n}\right)$ be a sequence of independent identically distributed (iid) random variables having common distribution function $F$. In EVT, we are interested in modeling the distribution of the maximum of the underlying random variables. Mathematically:

$$
M_{n}=\max \left(Y_{1}, Y_{2} \ldots, Y_{n}\right)
$$

If we assume that the distribution function $F_{Y}(y)$ is known, then we can write:

$$
\begin{aligned}
P\left(M_{n} \leq z\right) & =P\left(Y_{1} \leq z, Y_{2} \leq z \ldots, Y_{n} \leq z\right) \\
& =P\left(Y_{1} \leq z\right) \times P\left(Y_{2} \leq z\right) \times \ldots \times P\left(Y_{n} \leq z\right) \\
& =\left\{F_{Y}(z)\right\}^{n}
\end{aligned}
$$

In practice, the distribution function $F_{Y}(z)$ is unknown, and it can be either assumed to follow a particular family of distribution, or we can estimate the kernel density. The distribution function $F_{Y}(z)$ needs to be investigated when $\left(\mu_{n}, \sigma_{n}\right)$ exist, and $\mu_{n}, \sigma_{n} \in \mathbb{R}$, where each $\sigma_{n}>0$. Therefore,

$$
\lim _{n \rightarrow \infty} P\left(\frac{M_{n}-\mu_{n}}{\sigma_{n}} \leq z\right)=F_{Y}(z)
$$


The distribution function $F_{Y}(z)$ belongs to either the Fréchet, the Gumbel the or the Weibull family. In literature, there are numerous approaches exist to model the extreme values statistically. In this method, we focus on extreme events or the events of large-negative returns. In this regard, the two distributions: (i) GEV and (ii) GPD, are playing important roles.

The GEV is a continuous probability distribution use to model the tail events. The cumulative distribution function of the model is given by:

$$
F_{(\mu, \sigma, \xi)}(z)= \begin{cases}\exp \left\{-\left(1+\xi\left(\frac{z-\mu}{\sigma}\right)^{\frac{-1}{\xi}}\right)\right\} & , \xi \neq 0 \\ \exp \left\{-\exp \left(\frac{z-\mu}{\sigma}\right)\right\} & , \xi=0\end{cases}
$$

where $\mu \in \mathbb{R}$ is the location parameter, $\sigma>0$ is the scale parameter, and $\xi \in \mathbb{R}$ denotes the shape parameter. The parameter $\xi$ is also called the tail index. The values of $\xi$ classify three different types of extreme value distributions. For $\xi<0, \xi>0$, and $\xi=0$, the GEV converges to the negative Weibull, the Fréchet and the Gumbel distributions, respectively. These three distributions models followed the theory based on the Fisher-Tippet-Gnedento theorem (1928). The theorem states that there are only three types of distributions, the Fréchet, Gumbel and the Weibull, to model the maximum or minimum of the collection of random observations from the same distribution. In probability theory, the above-stated theorem is also called the three-type theorem. It is a general result in extreme value analysis. The theorem is similar to the central limit theorem for averages with finite variance. We use the GEV distribution often as an approximation to model the maxima of the long-finite sequence of random variables.

The generalized Pareto distribution (GPD) is a family of continuous probability distributions. The distribution has three parameters: $\mu \in \mathbb{R}$ is the location parameter, $\sigma>0$ is the scale parameter, and $\xi \in \mathbb{R}$ denotes the shape parameter. Pickands (1975) suggested that for $\xi \neq 0$, GPD results as a limiting distribution for the excess over thresholds when the parent distribution belongs to one of the extreme value distributions. The GPD reduces to the exponential distribution for $\mu=\xi=0$, and for $\xi<0(\xi>0)$, the GPD has a havier tail (lighter tail), respectively. Balkema and de Haan (1974) and Pickands (1975) show that for a large class of underlying distribution functions, the distribution function of the underlying sequence of iid random variables can be approximated by the GPD. The distribution function of the GPD is given by:

$$
F_{(\mu, \sigma, \xi)}(z)= \begin{cases}1-\left(1+\left(\frac{\xi(z-\mu)}{\sigma}\right)^{\frac{-1}{\xi}}\right) & , \xi \neq 0 \\ 1-\exp \left(-\frac{z-\mu}{\sigma}\right) & , \xi=0\end{cases}
$$


where $z \geq \mu$ for $\xi \geq 0$, and $\mu \leq z \leq \frac{\mu-\sigma}{\xi}$ when $\xi<0$. The parameter $\mu \in \mathbb{R}, \sigma$ is a scale parameter, and $\xi$ denotes the shape parameter.

We use three approaches to model the extreme values of PSX-100. We implement three approaches: block maxima, the peak-over-threshold (POT) and Poisson processes. To apply the block maxima method, a sufficiently large data set is usually required. The unavailability of the large data set consequently estimate the parameters of the distribution with great uncertainty. As we know, financial time series, particularly the stock returns, exhibit volatility clustering during unusual periods. Therefore, it may lead to an invalid selection of the block maxima values. As a result, risk measures such as Value-at-Risk (VaR) and expected-shortfall (ES) can be under-estimated.

Alternatively, another preferred approach, the peak-over-threshold (POT), takes large values of the sample, which exceed a certain threshold. The distribution function of values of exceedances is obtained by employing the GPD models. Unfortunately, a definite choice for the threshold level does not exist in the literature. For example, a low value of the threshold level may lead to a biased estimation. Similarly, a high value produces a fewer number of points for estimation. Usually, the mean excess function is used to obtain the threshold level, and the mean residual life plot results with a range of candidate values for the threshold level.

Financial returns exhibit fat-tail distributions. Therefore, common risk measures such as standard deviation can be misleading. Another most common risk measure is Value-at-Risk (VaR). It is used to evaluate the losses concerning market dynamics. Dedu and Ciumara (2010) used VaR risk measure to derive the restricted optimal retention in Stop-Loss reinsurance model. Dedu (2015) defined an extension of VaR, the Limited VaR, which better capture the tail behavior of the distribution model. The $\mathrm{VaR}$ is a quantile of distribution function exceeded with probability. Let $Y$ be a random variable. The VaR measure of the random variable $Y$ corresponding to the probability level $\alpha$ is given by:

or

$$
\operatorname{VaR}_{\alpha}(Y)=\inf \{y \in \mathbb{R} \mid P(Y<-y) \leq 1-\alpha\}
$$

$$
\operatorname{VaR}_{\alpha}(Y)=\inf \{y \in \mathbb{R}: F(y) \geq \alpha\},
$$

where $\alpha \in[0,1]$, and if $R_{t}$ denotes log-returns of the underlying time series then $\mathrm{VaR}_{\alpha}$ can be expressed as follows:

$$
\mathrm{VaR}_{\alpha}=\mu_{t}+\sigma_{t} \mathrm{q}^{-1}(\alpha)
$$

where $q$ denotes the quantile function of standardized log returns. Generally, $\alpha$ is taken to be 0.01 or 0.05 . For example, if we assume that 1 per cent five-days VaR 
is 1 dollar then, it means over the next five days, there is a 1 per cent chance of losing at least one dollar. See more details in McNeil et al. (2005).

Artzner et al. (1999) proposed an alternative risk measure of financial risk referred to as the expected shortfall (ES) or tail-VaR. It provides information on the entire tail of the distribution as a single measure. The ES measure is sub-additive and allows to compute expected loss when losses exceed VaR. Expected shortfall estimates the potential size of the loss exceeding. Let $Y$ is a random variable, then the expected shortfall is given by:

$$
\mathrm{E}\left(\mathrm{S}_{\alpha}\right)=\mathrm{E}\left(Y \mid Y \geq \operatorname{VaR}_{\alpha}(Y)\right)
$$

We fit GEV and GPD models to PSX-100 monthly largest negative returns. We use method of maximum likelihood (ML) and Probability-Weighted Moments (PWM) procedure. For more details on EVT, see Coles (2001).

\section{Data}

We begin the empirical applications with data analysis of the Pakistan Stock Exchange (PSX-100). We use logarithmic returns of the PSX-100. The logarithmic returns of all the underlying data series have been calculated by following $R_{t}=\ln \frac{x_{t}}{x_{t-1}}$, where $R_{t}$ denotes the daily closing return of the underlying stock. The data used in our empirical analysis consists of January 2001 to January 2021. We obtained the data from the Thomas Reuters' data stream. A preliminary examination of the descriptive statistics of the log-returns of PSX-100 showed that the return distributions have high kurtosis, positively skewed and heavier tails than a normal distribution. The Jarque-Bera statistics, Lilliefors, and Pearson ChiSquare test are significant at less than $1 \%$. Hence, there is a case for applying fattailed distributions rather than the normal distribution. Figure1 indicates that returns are not independent and identically distributed, and the volatility clustering phenomenon is present in the data. In Figure 2, the underlying returns have shown deviation from the normality assumption. Therefore, the rejection of the normally distributed returns justifies our choice of using the extreme value distributions. We examine the tail behavior of the PSX-100 returns by applying the EVT. For this purpose, we are considering the generalized extreme value distribution (GEV), generalized Pareto distribution (GPD) and mixtures of extreme value distributions. Figure 2 shows the QQ-plot of the empirical returns of PSX-100 versus the quantiles of the standard normal distribution. We observe significant deviation from the normal distribution both on the left and the right tail. 


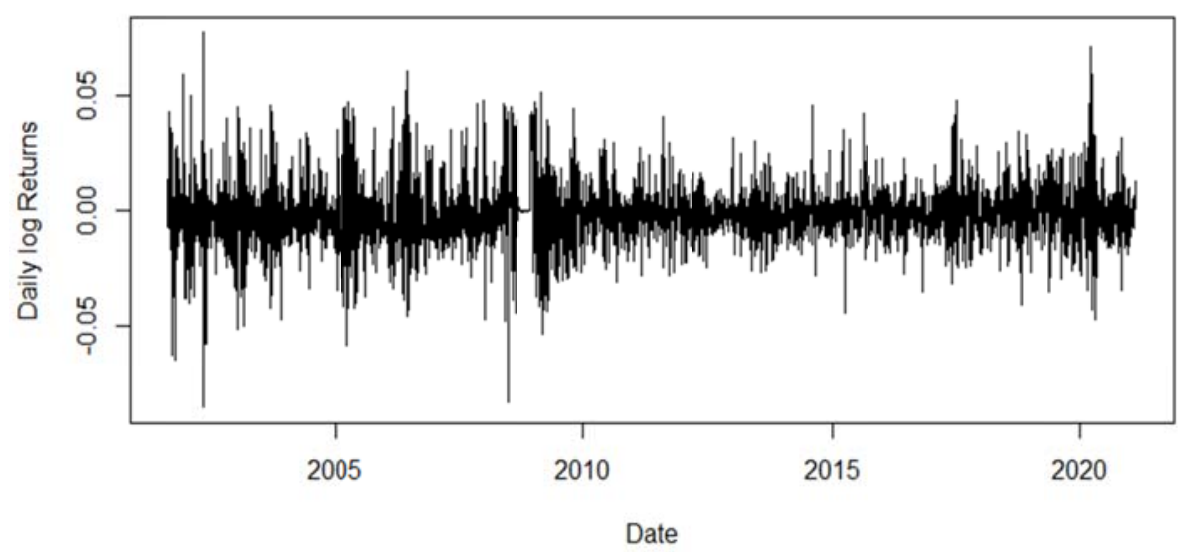

Figure 1. PSX-100 daily closing return series from Jan-2001 to Jan-2021

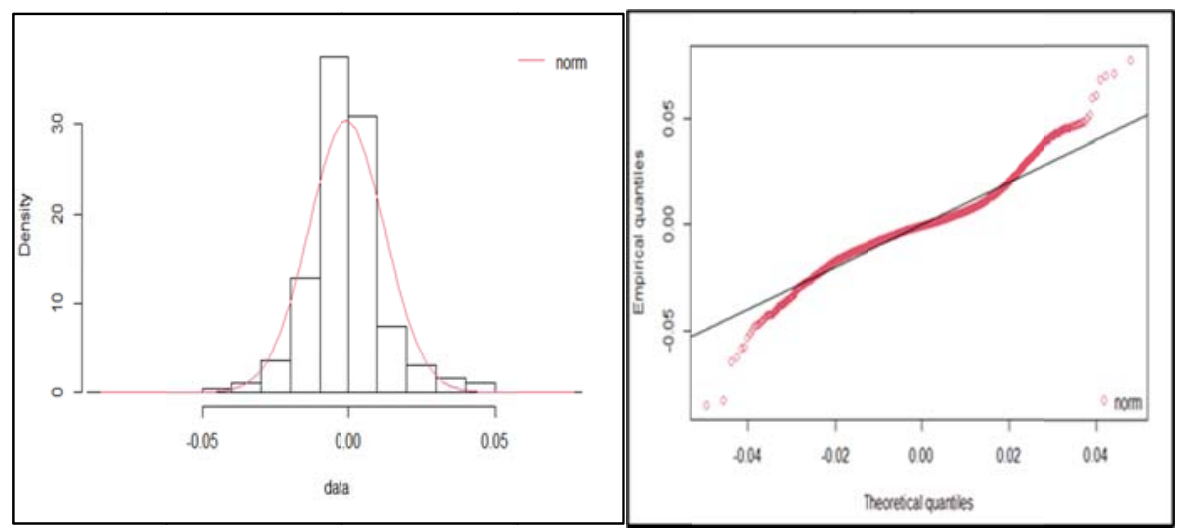

Figure 2. Histogram of PSX-100 daily closing return series with fitted normal distribution and Gaussian Kernel density estimation (KDE) and QQ Plot from Jan-2001 to Jan-2021

\section{Empirical Analysis}

We apply the EVT to PSX-100 data. We implement extreme value models using block maxima, POT, and point process to model the market risk.

In the first step, the block maxima method is applied to daily losses of PSX-100. We fit the GEV distribution to this data and present the inferences and diagnostic tests. For this purpose, we convert the daily returns to positive losses expresses in percentages. We extracted the monthly maxima of the data series. The data points extracted are shown in Figure 3. An increased volatility pattern has seen beyond 
the 100th observed loss point. Therefore, the assumption of identically distributed block maxima might lead to a violation. The parameter estimation results of GEV distribution followed by the maximum likelihood (ML) procedure are listed in Table 1.

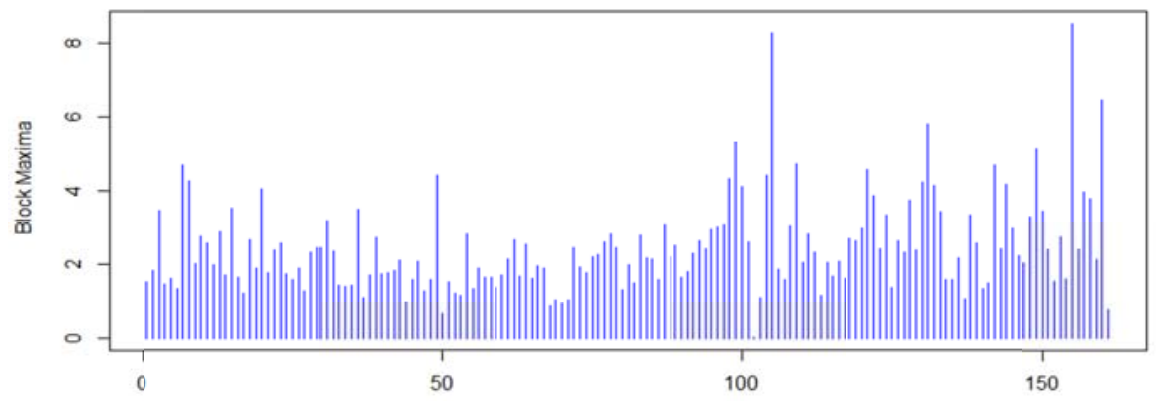

Figure 3. Monthly losses of PSX-100 daily returns from Jan 2001 to Jan 2021 We observe that all coefficients are significantly different from zero. The value of the shape parameter $\xi>0$, giving the heavy-tailed Fréchet case. We have also used the method of Probability-Weighted Moments (PWM) to estimate the parameters of GEV. The location parameter $\mu$, and the scale parameters $\sigma$, are close to the ML estimates. However, the estimate of the shape parameter $\xi=$ 0.1331 is non-negative and higher than the ML estimate- that is, the GEV is again of the Fréchet type. We observe in Figure 4 that the probability plot (PP) captured all the data points. However, the quantile plot (QQ) in the far-right tail has not followed few data points.
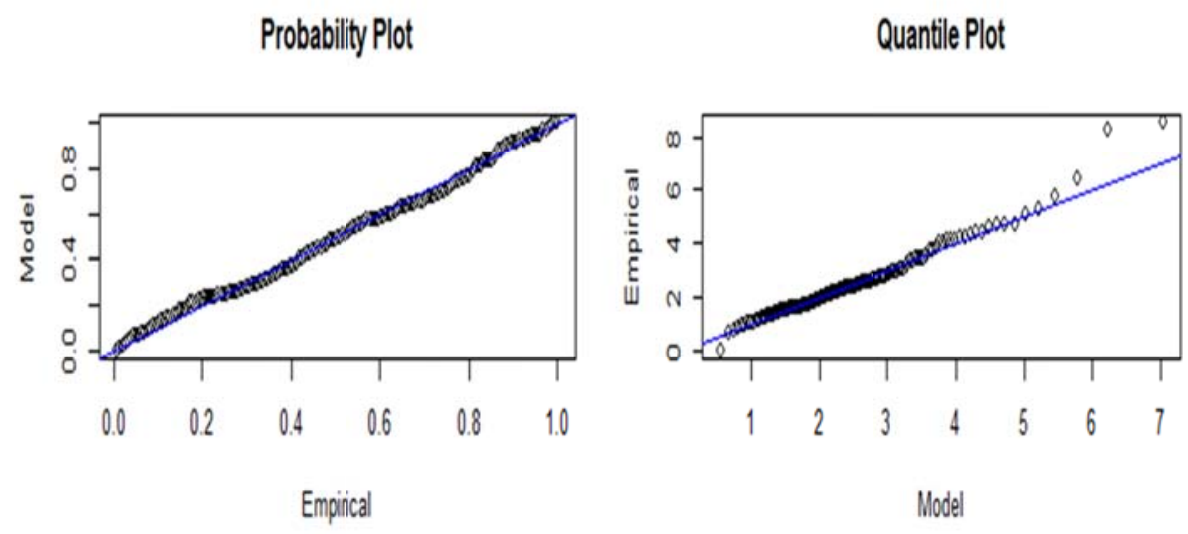

Figure 4. Diagnostic plots of GEV for PSX-100 
Extreme Value Analysis and Risk Assessment: A Case of Pakistan Stock Market

Table 1. Fitted GEV to block maxima of PSX-100

\begin{tabular}{|c|c|c|c|}
\hline \multicolumn{1}{|c|}{ GEV } & $\mu$ & $\sigma$ & $\xi$ \\
\hline Estimate (ML) & 1.8992288 & 0.8670732 & 0.0580542 \\
\hline Standard Error & 0.07475629 & 0.05407499 & 0.04568995 \\
\hline Estimate (PWM) & 1.8667601 & 0.8060077 & 0.1331637 \\
\hline
\end{tabular}

In the second step, we fit the GPD to daily losses of the PSX-100 by using the POT method. We use the approach of the mean residual life (MRL) plot to apply the POT method. A suitable value of the threshold level $u$ can be guided from Figure 6 with 95\% confidence intervals for the daily PSX-100 data. We observe that the graph appears to curve from $u=0.00$ to $u=0.04$, beyond which it is approximately linear until approximately $u=0.05$. Therefore, it seems reasonable to assume a daily loss as high as $4.5 \%$ as a threshold value, given that a linear relationship exists between the plotted thresholds and the mean excesses above this value. In theory, a threshold value slightly less or more than 4.5 might be beneficial. Consequently, it may provide uncertainty of the parameter estimates.

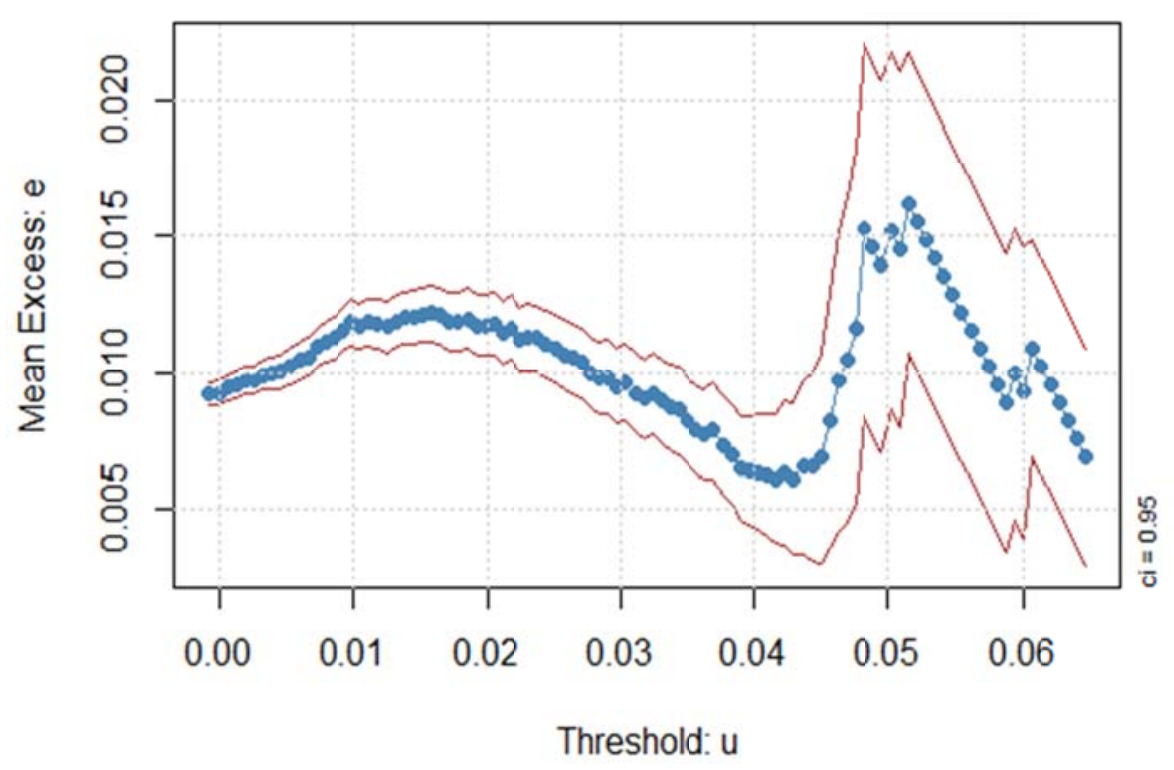

Figure 5. Mean Residual Life (MRL) plot for PSX-100 losses 
In the next step, we have fitted the GPD distribution to observed exceedances values. We assume that the exceedances are iid data points. The estimates of the fitted distribution and their standard errors have resulted with values of the shape parameter (standard error) $\xi=-0.0244(0.0164)$, respectively. The value of $\xi$ indicates the existence of a heavier tail. The scale parameter sigma is equal to 0.0091 with a standard error of 0.0002, and loglikelihood equals -9833.257 .

Now we investigate the goodness of fit of the fitted GPD model. For this purpose, we have shown the diagnostic plots in Figure 6. We present excess distribution plot, tail plot, residuals with the fitted ordinary least square line, and the QQ plot. We observe that the excess-distribution and tails plots both indicate a good fit of the GPD model to exceeded values. However, the tail plot has deviated from few data points at the far end. The least-square line of residuals shows the flat pattern, while the QQ plot has few deviations at the end of the plot.
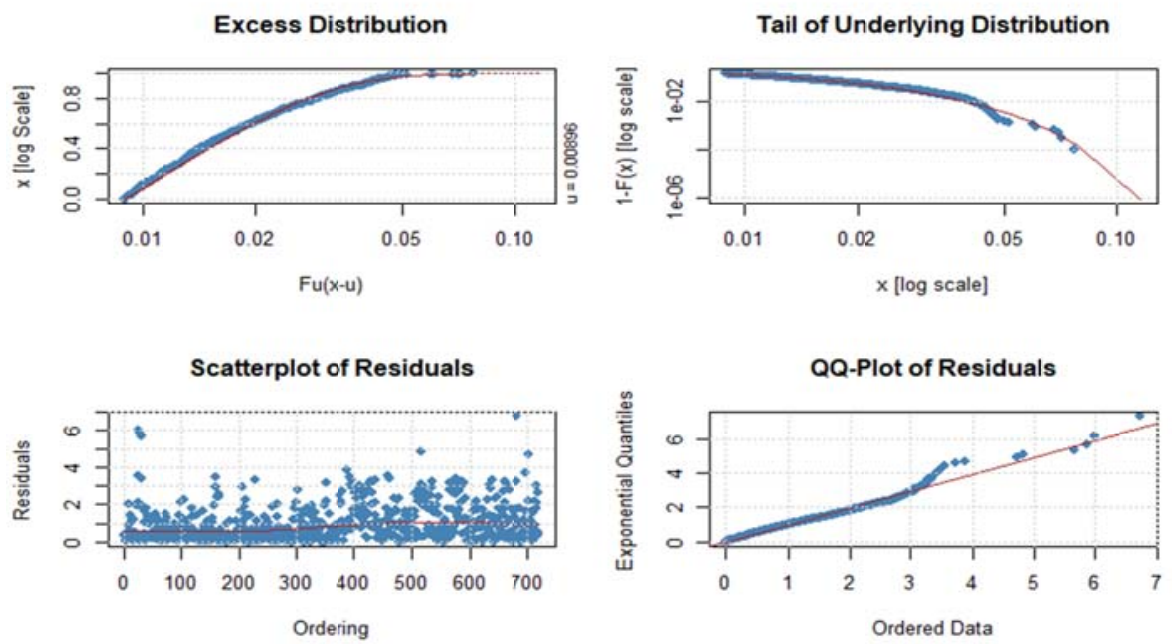

Figure 6. Diagnostic plots of the fitted GPD model.

Finally, the estimated values of $\mathrm{VaR}$ and $\mathrm{ES}$ are given in Table2. We compute these risk measures at 95\%, 99\% and 99.5\% level.

Table 2. Estimates of Risk Measures for PSX-100

\begin{tabular}{|c|c|c|}
\hline Confidence Level & VaR & ES \\
\hline $95 \%$ & 0.021889 & 0.032727 \\
\hline $99 \%$ & 0.039499 & 0.049433 \\
\hline $99.5 \%$ & 0.046623 & 0.056191 \\
\hline
\end{tabular}


In the third step, we use point processes to the daily-continuous losses of the PSX100 index. In the POT method, we assumed implicitly that the exceedances are iid data points. However, this assumption is barely well-founded for financial market returns, and extremes may tend to cluster in a stationary series. Therefore, the appropriateness of the models such as GEV and GPD needs to be validated. For example, in the GPD model, if threshold exceedances occur in the groups, it may lead to dependence among the observations. Therefore, the loglikelihood equation of the GPD model cannot be validated. For more precise mathematical development, see Leadbetter et al. (1983). We use the declustering approach to filter out the dependent observations. We recover the data for exceedances above the 95th percentile and retrieve the losses above the threshold. Therefore, for an iid Point process, time gaps between the exceedances will Poisson distributed. We have declustered the Point process data series into several periods, such as weekly, bi-weekly, monthly, bi-monthly, quarterly, and semi-annually. We recover the maximum from a cluster of exceedances to represent the extreme losses. We have fitted the GPD model to these series. We recover the maximum from a cluster of exceedances to represent the extreme losses. Table 3 shows the results of estimated parameters, loglikelihood and exceedance values. The standard errors of estimates are given in round brackets. The estimated values of the shape parameter behave differently for different declustered frequency periods.

Table 3 Parameter estimates of declustered GPD fitted model.

\begin{tabular}{|l|l|l|r|r|}
\hline Decluster Frequency & \multicolumn{1}{|c|}{$\xi$} & \multicolumn{1}{c|}{$\sigma$} & \multicolumn{1}{c|}{ Loglikelihood } & Exceedances \\
\hline Weekly & $\begin{array}{l}0.0536 \\
(0.0843)\end{array}$ & $\begin{array}{l}0.9658 \\
(0.1112)\end{array}$ & 165.0282 & 162 \\
\hline Bi-Weekly & $\begin{array}{l}0.0534 \\
(0.0985)\end{array}$ & $\begin{array}{l}1.0269 \\
(0.1381)\end{array}$ & 128.5131 & 119 \\
\hline Monthly & $\begin{array}{l}0.1163 \\
(0.1326)\end{array}$ & $\begin{array}{l}1.0119 \\
(0.1774)\end{array}$ & 84.61094 & 75 \\
\hline Bi-Monthly & $\begin{array}{l}0.0505 \\
(0.17)\end{array}$ & $\begin{array}{l}1.3363 \\
(0.2992)\end{array}$ & 62.99661 & 47 \\
\hline Quarterly & $\begin{array}{l}-0.0249 \\
(0.1921)\end{array}$ & $\begin{array}{l}1.6312 \\
(0.4228)\end{array}$ & 48.32616 & 33 \\
\hline Semi-annually & $\begin{array}{l}-0.0559 \\
(0.296)\end{array}$ & $\begin{array}{l}2.035 \\
(0.7879)\end{array}$ & 26.47207 & 16 \\
\hline
\end{tabular}

We can observe the departure from the iid assumption of observed losses employing several plots. Figure 7 shows diagnostic graphs of ACF. We have noticed exceedances do not occur randomly. The QQ plot depicts that the exceedances are clustered. Therefore, deviation from iid assumption has captured clearly. Figure 8 and Figure 9 shows QQ plots, excess distribution, and tail of the distribution plots of declustered periods of weekly, bi-weekly, monthly, bi- 
Muhammad Sheraz, Imran Nasir, Silvia Dedu

monthly, quarterly and semi-annually from top left to the right bottom, respectively. Table 4 shows the goodness of fit $(\mathrm{GoF})$ results for both the GEV and GPD models. The small p-values of the GPD suggest accepting it as the best-fitted model.

\begin{tabular}{|c|c|c|c|c|}
\hline GoF Test & \multicolumn{2}{|c|}{ GEV Distribution } & \multicolumn{2}{c|}{ GPD Distribution } \\
\hline & Statistics & P-Value & Statistics & P-Value \\
\hline Cramer-von Misses & 0.067 & 0.30617 & 0.2272 & 0.00246 \\
\hline Anderson-Darling & 0.4649 & 0.25414 & 1.3749 & 0.00147 \\
\hline
\end{tabular}

Weekly De-clustered Loss

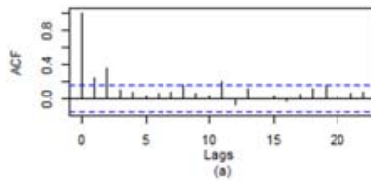

Bi-Monthly De-clustered Loss

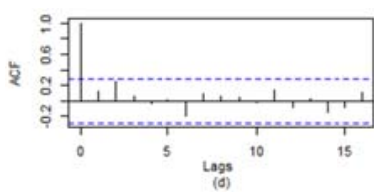

Bi.Weekly De-clustered Loss

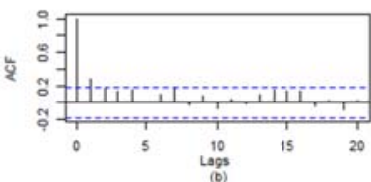

Quaterly De-clustered Loss

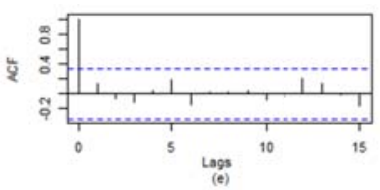

Monthly De-clustered Loss

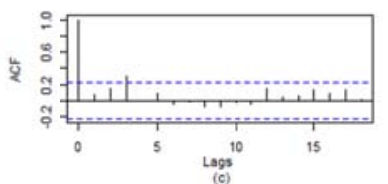

Semi-annually De-clustered Loss

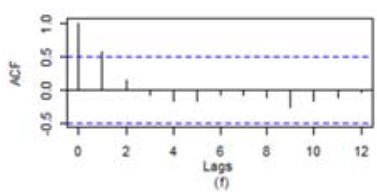

Figure 7. Diagnostic ACF plots of the fitted GPD model for declustered observations

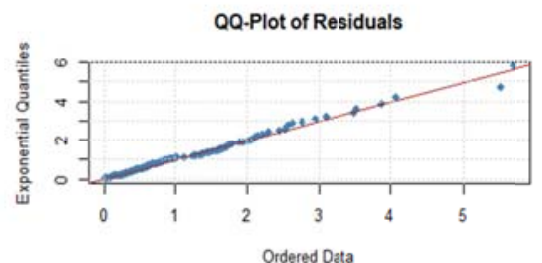

QQ-Plot of Residuals

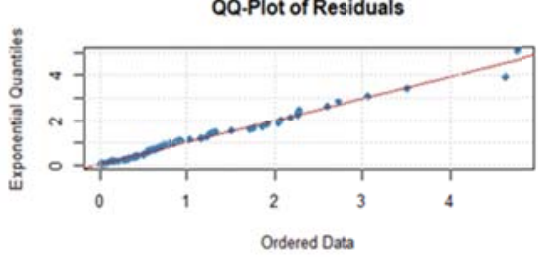

QQ-Plot of Residuals

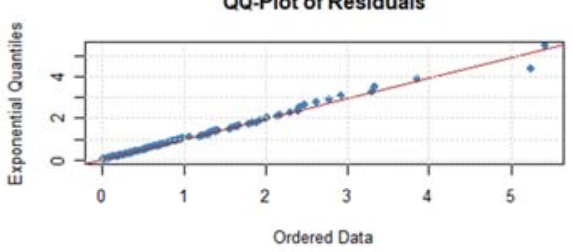

QQ-Plot of Residuals

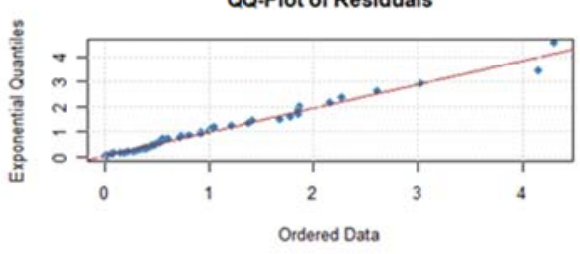


Extreme Value Analysis and Risk Assessment: A Case of Pakistan Stock Market
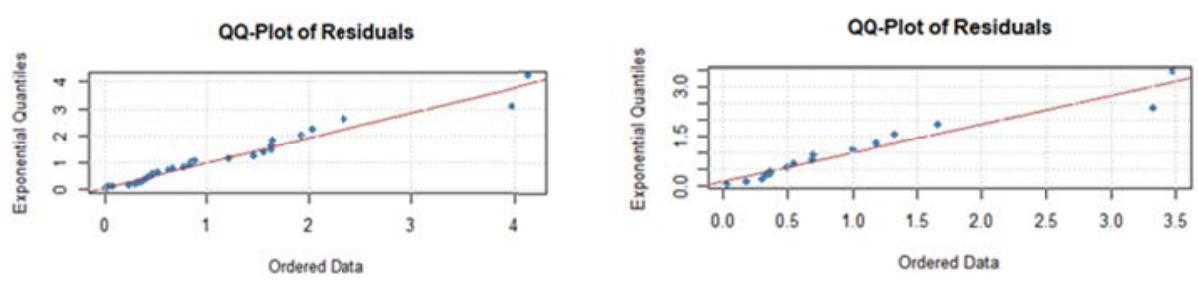

Figure 8. QQ plots of GPD fitted model for declustered observations.
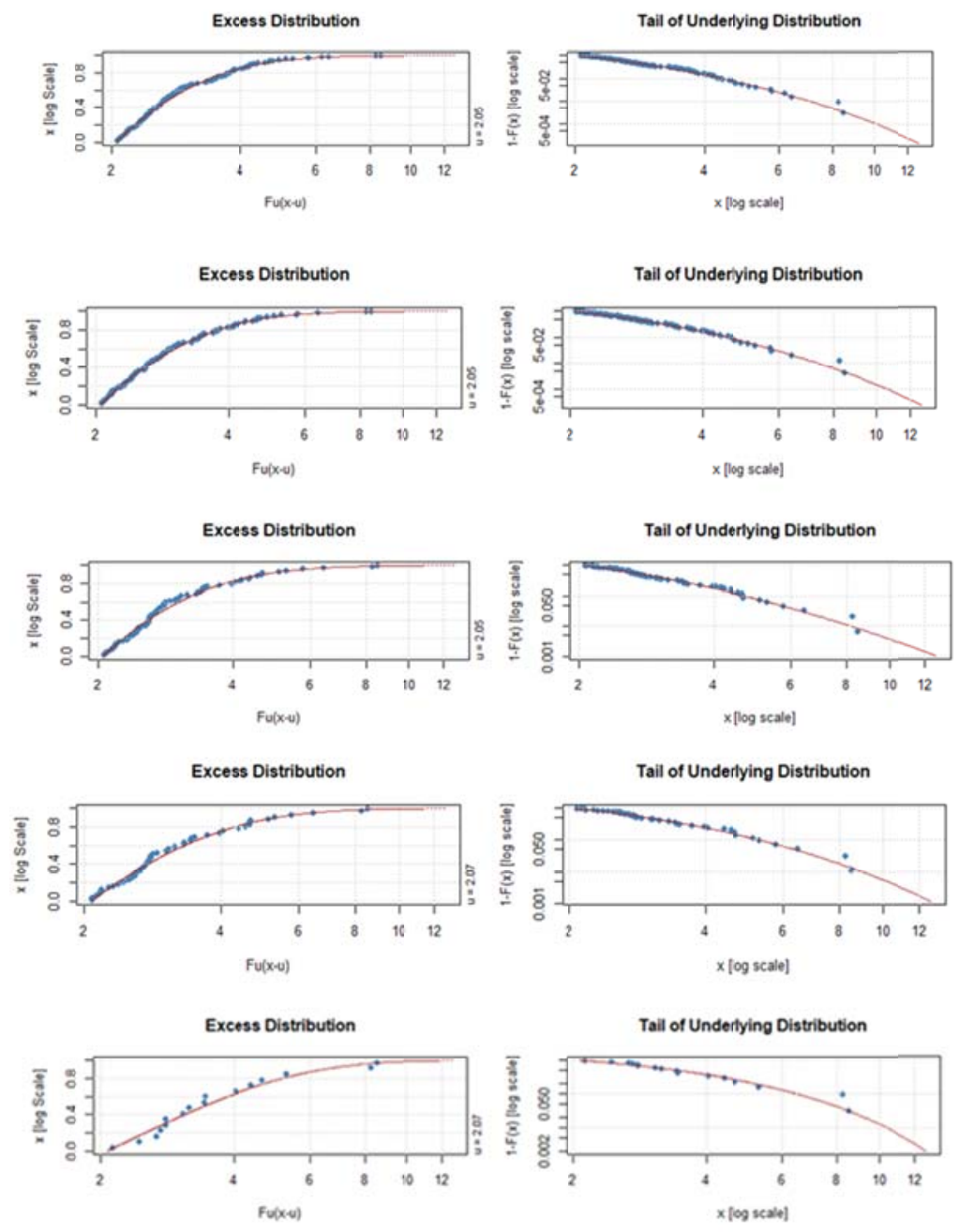

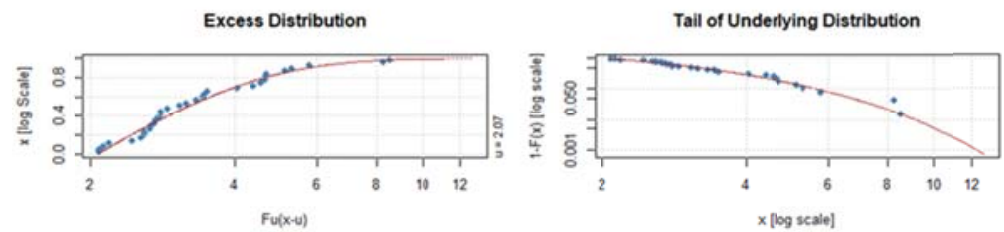

Figure 9. Plots of excess distribution and tail of the GPD fitted model for declustered observations.

\section{Conclusions}

The Pakistani stock market (PSX-100) exhibit a high degree of volatility and, it is sensitive to reacts quickly to political news, government policies and media developed stories. Consequently, the risky nature of the market provides the basis to implement the EVT. The main aim of the present study was to evaluate different statistical methods used for the analysis of extreme events of stock returns. For this purpose, daily closing returns of PSX-100 for twenty years data have been analyzed. The data was organized into new data sets, and we characterized the risk properties of the financial market. The GEV distribution fits as a Fréchet type to the block maxima data set. We estimated the parameters of the employed model by using the methods of MLE and PWM. In the second step, we used the POT method to fit the GPD model. However, the estimated parameters values and the diagnostic plots and goodness of fit methods show that the GPD model performed better. We have also applied the GPD model to declustered periods by using the point processes method. We investigate that the sensitivity of the model's parameters is high, and it depends on the number of declustered periods. The estimated values by this method become unauthentic if the number of observations in a declustered period is less. We examine that the returns distribution of PSX-100 is fatter than the normal distribution. Therefore, GEV and GPD models perform well in modelling extreme events, and risk measures such as VaR and ES in this regard can be used effectively. Finally, this empirical study characterized the emerging south-Asian stock market, PSX-100, to model the extreme events.

ACKNOWLEDGEMENT: This work was supported by a grant of the Romanian Ministry of Education and Research, CNCS - UEFISCDI, project number PN-III-P4-ID-PCE-2020-1112, within PNCDI III. 
Extreme Value Analysis and Risk Assessment: A Case of Pakistan Stock Market

\section{REFERENCES}

[1] Balkema, AA., Haan. L. (1974), Residual Lifetime at Great Age. The Annals of Probability, 2(5): 792-804;

[2] Bortkiewicz, L.V. (1922), Variationsbreite und mittlerer Fehler. Sitzungsber. Berli. Math. Gess, 3(21): 3-11;

[3] Bozovic, M., Totic, S. (2015), Tail Risk in Emerging Markets of Southeastern Europe. Applied Economics, 48(19): 1785-1798;

[4] Beirlant J., Goegebeur Y., Segers J., Teugels J. (2004), Statistics of Extremes: Theory and Applications. John Wiley \& Sons, Chichester, England;

[5] Coles, S. (2001), An Introduction to Statistical Modeling of Extreme Values. Springer-Verlag London limited;

[6] Dedu, S. (2015), A New Risk Assessment and Optimization Model with Applications to Insurance; Economic Computation and Economic Cybernetics Studies and Research; ASE Publishing; 49, 2, 129-142;

[7] Dedu, S., Ciumara, R. (2010), Restricted Optimal Retention in Stop-loss Reinsurance under Var and CTE Risk Measures, Proceedings of the Romanian Academy, 11, 3, 213-217, 2010;

[8] Djakovic, V., Andjelic, G., Borocki, J. (2011), Performance of Extreme Value Theory in Emerging Markets. An empirical treatment. African Journal of Business Management, 5(2): 340-369;

[9] Gencay, R., Selcuk, F. (2004), Extreme Value Theory and Value-At-Risk: Relative Performance in Emerging Markets. International Journal of Forecasting, 20: 287-303;

[10] Goncu, A., Akgul, A.K., Imamoglu, O., Tiryakioglu, M. (2012), An Analysis of the Extreme Returns Distribution: The Case of the Istanbul Stock Exchange. Applied Financial Economics, 22(19), 723-732;

[11] Fisher, R., Tippett L.H.C. (1928), Limiting Form of the Frequency Distribution of the Largest or Smallest Member of a Sample. Mathematical Proceedings of the Cambridge Philosophical Society, 24: 180-190;

[12] Hill, B. M. (1975), A Simple General Approach to Inference about the Tail of a Distribution. Annals of Statistics, 3: 1163-1174;

[13] Leadbetter M., Lindgren G., Rootzén, H. (1983), Extremes and Related Properties of Random Sequences and Series. Springer-Verlag, New York; [14] Mandelbrot, B. (1963), The Variation of Certain Speculative Prices. Journal of Business, 36:394-419;

[15] Melevergne, Y., Pisarenko, V., Sornette, D. (2006), On the Power of Generalized Extreme Value (GEV) and Generalized Pareto Distribution (GPD) Estimator for Empirical Distributions of Stock Returns. Applied Financial Economics, 16(3): 271-289;

[16] McNeil A., Frey R., Embrechts, P. (2005), Quantitative Risk Management: Concepts, Techniques and Tools. Princeton University Press, Princeton, NJ; 
Muhammad Sheraz, Imran Nasir, Silvia Dedu

[17] Pickands, J. (1975), Statistical Inference Using Extreme Order Statistics. The Annals of Statistics, 3(1): 119-131;

[18] Preda, V., Dedu, S., Sheraz, M. (2016), Second Order Entropy Approach for Risk Models Involving Truncation and Censoring; Proceedings of the Romanian Academy, 17, 3, 195-202;

[19] Rama, C. (2001), Empirical Properties of Asset Return: Stylized Facts and Statistical Issues. Quantitative Finance, 1:23-236;

[20] Sheraz, M., Nasir, I. (2021), Information-Theoretic Measures and

Modeling Stock Market Volatility: A Comparative Approach. Risks, 9, 5: 89. https://doi.org/10.3390/risks9050089;

[21] Sheraz, M., Preda, V., Dedu, S. (2020), Non-extensive Minimal Entropy Martingale Measures and Semi-Markov Regime Switching Interest Rate Modeling. AIMS Mathematics, 5, 1, 300-310;

[22] Sheraz, M., Dedu, S. (2020), Bitcoin Cash: Stochastic Models of Fat-tail Returns and Risk-modeling. Economic Computation and Economic Cybernetics Studies and Research; ASE Publishing, 54(3): 43-58;

[23] Sing, A.K., Allen, D.E., Powell, R.J. (2017), Tail Dependence Analysis of Stock Markets Using Extreme Value Theory. Applied Economics, 49(45): 45884599;

[24] Uppal, Y.J., Mudakkar, M.R. (2014), Challenges in the Application of Extreme Value Theory in Emerging Markets: A Case Study of Pakistan. In Risk Management Post Financial Crisis: A Period of Monetary Easing, 96:417-437. 